\title{
Circuit-Specific Plasticity of Callosal Inputs Underlies Cortical Takeover
}

\author{
${ }^{\circledR}$ Emily Petrus, ${ }^{1}$ Sarah Dembling, ${ }^{1}{ }^{\circledR}$ Ted Usdin, ${ }^{2}$ John T.R. Isaac, ${ }^{3}$ and ${ }^{\circledR}$ Alan P. Koretsky ${ }^{1}$ \\ ${ }^{1}$ Laboratory of Functional and Molecular Imaging, National Institute of Neurological Disorders and Stroke, National Institutes of Health, Bethesda, \\ Maryland 20892, ${ }^{2}$ Systems Neuroscience Imaging Resource, National Institute of Mental Health, Bethesda, Maryland 20892, and ${ }^{3}$ Janssen \\ Neuroscience, J\&J Innovations, London W1G 0BG, United Kingdom
}

Injury induces synaptic, circuit, and systems reorganization. After unilateral amputation or stroke, this functional loss disrupts the interhemispheric interaction between intact and deprived somatomotor cortices to recruit deprived cortex in response to intact limb stimulation. This recruitment has been implicated in enhanced intact sensory function. In other patients, maladaptive consequences such as phantom limb pain can occur. We used unilateral whisker denervation in male and female mice to detect circuitry alterations underlying interhemispheric cortical reorganization. Enhanced synaptic strength from the intact cortex via the corpus callosum (CC) onto deep neurons in deprived primary somatosensory barrel cortex $(\mathrm{S} 1 \mathrm{BC})$ has previously been detected. It was hypothesized that specificity in this plasticity may depend on to which area these neurons projected. Increased connectivity to somatomotor areas such as contralateral S1BC, primary motor cortex (M1) and secondary somatosensory cortex (S2) may underlie beneficial adaptations, while increased connectivity to pain areas like anterior cingulate cortex (ACC) might underlie maladaptive pain phenotypes. Neurons from the deprived S1BC that project to intact S1BC were hyperexcitable, had stronger responses and reduced inhibitory input to CC stimulation. M1-projecting neurons also showed increases in excitability and CC input strength that was offset with enhanced inhibition. S2 and ACC-projecting neurons showed no changes in excitability or CC input. These results demonstrate that subgroups of output neurons undergo dramatic and specific plasticity after peripheral injury. The changes in S1BC-projecting neurons likely underlie enhanced reciprocal connectivity of $\mathrm{S} 1 \mathrm{BC}$ after unilateral deprivation consistent with the model that interhemispheric takeover supports intact whisker processing.

Key words: cell specificity; corpus callosum; injury plasticity; interhemispheric plasticity; somatosensory system

Significance Statement

Amputation, peripheral injury, and stroke patients experience widespread alterations in neural activity after sensory loss. A hallmark of this reorganization is the recruitment of deprived cortical space which likely aids processing and thus enhances performance on intact sensory systems. Conversely, this recruitment of deprived cortical space has been hypothesized to underlie phenotypes like phantom limb pain and hinder recovery. A mouse model of unilateral denervation detected remarkable specificity in alterations in the somatomotor circuit. These changes underlie increased reciprocal connectivity between intact and deprived cortical hemispheres. This increased connectivity may help explain the enhanced intact sensory processing detected in humans.

Received May 6, 2020; revised June 13, 2020; accepted Aug. 21, 2020

Author contributions: E.P., J.T.R.I., and A.P.K. designed research; E.P., S.D., and T.U. performed research; T.U. contributed unpublished reagents/analytic tools; E.P. and S.D. analyzed data; E.P. wrote the paper. The authors declare no competing financial interests.

This work was supported by the Intramural Research Program of the National Institutes of Health, National Institute of Neurological Disorders and Stroke. We thank Ray Fields for virus production; Jonathan Kuo, Sarah Williams, and Kaji Sheth for image processing assistance, Jeff Diamond for helpful discussions; and Kathy Sharer and Nadia Bouraoud for animal assistance.

Correspondence should be addressed to Emily Petrus at emily.petrus@nih.gov.

https://doi.org/10.1523/JNEUROSCI.1056-20.2020

Copyright $\odot 2020$ Petrus et al.

This is an open-access article distributed under the terms of the Creative Commons Attribution License Creative Commons Attribution 4.0 International, which permits unrestricted use, distribution and reproduction in any medium provided that the original work is properly attributed.

\section{Introduction}

Central or peripheral injury drives widespread cortical remodeling (Garraghty and Kaas, 1991; Navarro et al., 2007). Amputees experience a recruitment of the deprived sensory/motor cortex leading to bilateral responses to intact limb stimulation (Lotze et al., 2001; MacIver et al., 2008; Simões et al., 2012). The behavioral importance and synaptic mechanisms responsible for these changes are unknown. For example, some studies show that patients experience beneficial adaptations which enhance prosthetic use or increase intact limb sensitivity (Chen et al., 2013a; Templeton et al., 2018). Other studies indicate that remodeling may be related to phantom limb pain (Flor et al., 2006); however, 
this cortical remodeling is not consistently observed in phantom limb (Makin et al., 2013, 2015). A rodent model of unilateral whisker denervation (infraorbital nerve transection: ION-X) mimics some of the changes detected in humans; for example there is potentiation of the pathway into intact primary somatosensory barrel cortex (S1BC) and task-evoked recruitment of deprived S1BC during intact whisker stimulation (Yu et al., 2012; Petrus et al., 2019). The recruitment of deprived S1BC is likely because of potentiation of the corpus callosum (CC) inputs from intact S1BC to deprived S1BC, which was so strong to L5 neurons that long-term potentiation (LTP) was occluded (Petrus et al., 2019). Interestingly, this plasticity was not found in L2/3 neurons, demonstrating specificity to deep layer neurons. Because deep cortical neurons often represent output cells for the sensory cortical column (Douglas and Martin, 2004), it was hypothesized that the plasticity may be specific to subsets of neurons based on the downstream brain regions they target. The CC input is believed to be relatively uniform as are cortically projecting L5 principal neurons. However, the differential plasticity of the CC restricted to the deep neurons in deprived S1BC leads to the hypothesis that the plasticity because of ION-X is highly specific.

Throughout the brain, there are examples of input and output specificity, where neurons restrict changes to specific inputs, or provide altered outputs (Larsen and Sjöström, 2015; Rawson et al., 2017). Efforts to describe these changes are limited to alterations in input or output specificity between interneurons and principal cells (Trouche et al., 2013; Lu et al., 2014; Lee et al., 2016) or laminar-specific adaptations (Koester and Johnston, 2005; Sjöström and Häusser, 2006). L5 neurons have been categorized by their electrophysiological properties (Shai et al., 2015), connectivity (Hattox and Nelson, 2007), morphology (Oswald et al., 2013), location (Lefort and Petersen, 2017), and gene expression (Tasic et al., 2016; Clare et al., 2018). These cortical output neurons send long-range projections throughout the brain and are thus optimally situated to integrate columnar information (Koester and Johnston, 2005; Sjöström and Häusser, 2006; Baker et al., 2018) and effect widespread adaptations (Grillner et al., 2005; Feldmeyer, 2012; Petersen, 2019). It is not known whether a uniform input (like the CC) can produce restricted plasticity depending on output neuron connectivity. In the present work, CC input onto L5 neurons projecting to output regions involved in whisker sensation and movement [primary motor cortex (M1), intact S1BC, secondary somatosensory cortex (S2)] or pain perception [anterior cingulate cortex (ACC) and S2] were studied. Retrograde viruses (rAAVs)-labeled distinct cell populations that project from the deprived S1BC to contralateral S1BC, M1, S2, and ACC. After ION-X, reciprocally connected S1BC neurons were hyperexcitable, had stronger responses to CC stimulation and less CC mediated inhibition. Cells projecting to $\mathrm{M} 1$ were also hyperexcitable and had stronger CC synapses but a concurrent increase in inhibition resulted in an unchanged total callosal response. Cells projecting to S2 and ACC were unchanged in any parameters measured. It was demonstrated that L5 output neurons experience plasticity to a uniform input (the CC) depending on their output targets. The increase in bilateral connectivity supports the hypothesis that the intact S1BC colonizes the deprived S1BC after unilateral sensory loss to aid intact whisker processing.

\section{Materials and Methods}

\section{Animals}

All procedures were approved by the National Institutes of Health Animal Care and Use Committee (ACUC) under protocol 1160, and facilities are accredited by the Association for Assessment and
Accreditation of Laboratory Animal Care (AAALAC). C57Bl/6 (RRID: IMSR_JAX:000664) and $\mathrm{PV}^{+}$cre (RRID: IMSR_JAX:008069) mice were bred in house. Both mouse lines were originally purchased from The Jackson Laboratory. Equal gender distributions were used throughout the study. No significant differences in results were detected between genders. Littermates were housed one to four per cage with food and water ad libitum on a 12/12 h light/dark cycle.

\section{Surgery}

Stereotaxic surgery

At postnatal day (P)21, pups were weaned, anesthetized with 1-3\% isoflurane mixed with $\mathrm{O}_{2}$, and unilaterally injected (right side) with $500 \mathrm{nl}$ of a virus encoding for channel rhodopsin (ChR2): AAV2/9.hSynapsin. hChR2(H134R)-EYFP.WPRE.hGH, RRID: Addgene_26973 (Penn Vector Core, University of Pennsylvania). Animals received the viral injection into S1BC. Coordinates from bregma for S1BC were: anterior/posterior (AP) -1.0, medial/lateral (ML) 3.0, and depth from pia $0.5 \mathrm{~mm}$. Mice recovered on a heated surface and received postoperative care including three once daily subcutaneous saline/ketaprofen solution injections.

Retrograde viral injections (rAAVs) were injected four to six weeks after initial stereotaxic injections and occurred on the same surgery day as sham/ION-X surgery. Injection locations were: left S1BC (coordinates: $\mathrm{AP}-1.0, \mathrm{M}-3.0$, depth $0.5 \mathrm{~mm}$ ), right $\mathrm{M} 1$ (coordinates: AP 1.1, $\mathrm{M} 1.8$, depth 0.5), right S2 (coordinates: AP -1.0, M 4.0, depth 0.9), or right ACC (coordinates: AP 1.34, M 0.4, depth 1.0). rAAV injections occurred one at a time for electrophysiology experiments using only the mCherry fluorophore for optimal visibility. Quadruple injections used different colors for each injection: S1BC: GFP, M1: mOrange, S2: mAmetrine, ACC: mCherry. GFP and mCherry retrograde viruses were tailored in the NINDS viral production core from Addgene plasmids: pAAV-CAG-eGFP, and pAAV-CAG-mCherry, respectively. mOrange, mAmetrine, and floxed red were made from Addgene plasmids: RRID:Addgene_54680, RRID:Addgene_56542, RRID:Addgene_ 20299, respectively.

\section{Sham/ION-X surgery}

Four to six weeks after stereotaxic viral injection mice received a sham or ION-X surgery. Mice were weighed and received an intraperitoneal injection of a ketamine/xylazine cocktail (ketamine $80 \mathrm{mg} / \mathrm{kg} / \mathrm{xylazine}$ $10 \mathrm{mg} / \mathrm{kg}$ ). Once the absence of the hindpaw pinch reflex was observed, whiskers were shaved unilaterally and an incision caudal to the whisker pad was made to visualize the bundle of infraorbital nerves. The sham animals' surgery finished at this step; the incision was closed with one suture and tissue glue (Tissuemend II, Veterinary Product Laboratories). The nerve bundle was cut with scissors in ION-X experimental animals, and the incision was closed in the same manner as the sham group. Animals then received an intraperitoneal injection of Antisedan (1 mg/ $\mathrm{kg}$; Atipamezole, Zoetis) to reverse the anesthesia. Animals remained on a heated surface and returned to the animal facility after they were fully ambulatory, usually after 10-30 $\mathrm{min}$.

\section{Electrophysiology}

\section{Acute slice preparation}

Two weeks after ION-X or sham surgeries acute slices were made for whole-cell electrophysiological recordings. Mice were anesthetized using isoflurane vapors $\left(5 \%\right.$ mixed with $\mathrm{O}_{2}$ ) until the absence of the corneal reflex was observed. The brain was quickly dissected and immersed in ice-cold dissection buffer ( $80 \mathrm{~mm} \mathrm{NaCl}, 3.5 \mathrm{~mm} \mathrm{KCl}, 1.25 \mathrm{~mm} \mathrm{H}_{2} \mathrm{PO}_{4}, 25$ mм $\mathrm{NaHCO}_{3}, 4.5 \mathrm{~mm} \mathrm{MgSO}_{4}, 0.5 \mathrm{~mm} \mathrm{CaCl}_{2}, 10 \mathrm{~mm}$ glucose, and $90 \mathrm{~mm}$ sucrose), which was bubbled continuously with a $95 \% \mathrm{O}_{2} / 5 \% \mathrm{CO}_{2}$ gas mixture. Brain blocks containing primary somatosensory cortex were dissected and coronally sectioned into $300-\mu$ m-thick slices using a Leica VT1000S vibratome (Leica Biosystems Inc.). Slices were incubated for $30 \mathrm{~min}$ at $35^{\circ} \mathrm{C}$ and then returned to room temperature for a minimum of 30 additional minutes before recordings began.

Slices were transferred to a submersion-style recording chamber mounted on a fixed stage (Sutter Instruments Company) with an upright Nikon Eclipse FN1 microscope (Nikon Instruments) and illuminated with oblique infrared (IR) illumination. Recordings were all performed 
in artificial CSF (ACSF; $124 \mathrm{~mm} \mathrm{NaCl}, 5 \mathrm{~mm} \mathrm{KCl}, 1.25 \mathrm{~mm}$ $\mathrm{NaH}_{2} \mathrm{PO}_{4} \cdot \mathrm{H}_{2} \mathrm{O}, 26 \mathrm{~mm} \mathrm{NaHCO}, 10 \mathrm{~mm}$ dextrose, $2.5 \mathrm{~mm} \mathrm{CaCl}_{2}$, and $1.5 \mathrm{~mm} \mathrm{MgCl}$ ) bubbled with $95 \% \mathrm{O}_{2} / 5 \% \mathrm{CO}_{2}$ at $30^{\circ} \mathrm{C}$ unless otherwise indicated. The ACSF was continually perfused at a rate of $2 \mathrm{ml} / \mathrm{min}$. Voltage clamp experiments used Cs-gluconate internal solution, which contained the following: $130 \mathrm{~mm}$ Cs-gluconate, $8 \mathrm{~mm} \mathrm{KCl}, 1 \mathrm{~mm}$ EGTA, 10 mм HEPES, 4 mм ATP, and 5 mм QX-314; pH 7.3, 285295 mOsm. Current-clamp experiments used K-gluconate internal solution, which contained the following: $130 \mathrm{~mm}$ K-gluconate, $10 \mathrm{~mm}$ $\mathrm{KCl}, 0.2$ mм EGTA, $10 \mathrm{~mm}$ HEPES, 4 mм MgATP, $0.5 \mathrm{~mm}$ NaGTP, and $10 \mathrm{~mm}$ Naphosphocreatine; pH 7.3, 280-290 mOsm). Cells with an access resistance higher than $25 \mathrm{M} \Omega$ and input resistance lower than $100 \mathrm{M} \Omega$ were discarded. Cells which had more than a $20 \%$ fluctuation in these values for long-term recordings were excluded from analysis. A maximum of three cells per animal, one cell per slice were recorded for each dataset.

An axon patch-clamp amplifier 700B (Molecular Devices) was used for voltage clamp recordings. Data were acquired through pClamp10 and analyzed with Clampfit 10.4 software (Molecular Devices).

\section{Connection probability of callosally targeted principal cells}

L5 neurons labeled with rAAV-mCherry expression in deprived S1BC were visually identified and patched for whole-cell recordings. ChR2 was activated using a 455-nm light emitting diode (LED) DC2100, illuminated through the $40 \times$ objective lens and controlled by a digital stimulator (Cygnus DG4000A), both from ThorLabs. Cells which responded to LED stimulation were counted as responding to callosal inputs, while those with no response, even to maximum LED intensity, were counted as not responding. The probability of $\mathrm{CC}$ response varied between groups, with reciprocally connected S1BC neurons and S2 output neurons exhibiting the greatest probability of CC response (72\% and 70\%, respectively), while $\mathrm{M} 1$ and $\mathrm{ACC}$ output neurons were the less likely to be targeted by the CC ( $60 \%$ and $35 \%$, respectively). Less than $10 \%$ of neurons in any group were driven to spike with LED stimulation of ChR2-expressing CC fibers. Subsequent experiments consistently verified CC targeting of rAAV-labeled neurons before recordings.

\section{Intrinsic excitability measurements}

These experiments were performed as described previously (Petrus and Lee, 2014). Pipettes with tip resistances of 4- to 6-M $\Omega$ resistance were filled with K-gluconate internal solution and after verifying CC-mediated responses, $10 \mu \mathrm{M}$ NBQX, $100 \mu \mathrm{M}$ APV and $50 \mu \mathrm{M}$ bicuculline were added to ACSF to block AMPA, NMDA, and $\mathrm{GABA}_{\mathrm{A}}$ receptors, respectively. L5 principal neurons were recorded in current clamp with current injected to maintain the cells at $-70 \mathrm{mV}$. Current pulses of $800-\mathrm{ms}$ duration were injected at 10 -s intervals with increasing amplitudes of 20-pA steps for 15 repetitions (starting with $0 \mathrm{pA}$, maximum injection of $300 \mathrm{pA}$ ). From the data collected, the rheobase (minimum current needed to produce a single action potential) was calculated for sham and deprived S1BC output L5 neurons. The resting membrane potential and input resistances were also measured to determine baseline excitability for these neurons. Cell properties are listed in Extended Data Figure 1-1.

\section{$\mathrm{Sr}^{2+}$ mEPSCs}

Methods are similar to those described previously (Petrus et al., 2014). AMPAR-mediated $\mathrm{Sr}^{2+}$ mEPSCs were isolated pharmacologically with $20 \mu \mathrm{M}$ bicuculline and $100 \mu \mathrm{M}$ DL-2-amino-5 phosphonopentanoic acid (DL-APV). ACSF was as described previously; however, there was $0 \mathrm{~mm}$ $\mathrm{CaCl}_{2}, 4 \mathrm{mM} \mathrm{MgCl}_{2}$, and $4 \mathrm{~mm} \mathrm{SrCl}_{2}$. rAAV-labeled L5 neurons in deprived S1BC were identified visually and patched using whole-cell patch pipettes with tip resistances between 4 and $6 \mathrm{M} \Omega$, which was filled with Cs-gluconate internal solution.

The minimum LED intensity was used to elicit reliable responses and desynchronized $\mathrm{Sr}^{2+}$ mEPSCs ChR2-mediated responses were obtained. Cells were held at $-70 \mathrm{mV}$ and recorded for a minimum of $10 \mathrm{~min}$. Data were acquired every $10 \mathrm{~s}$ for a duration of $1200 \mathrm{~ms}$, which included a 500-ms duration prestimulation, a 500-ms duration postLED illumination, and a $100-\mathrm{ms}$ seal test pulse. The 400 -ms window preStim was used to quantify the spontaneous events (preLED) and the 400-ms window after stimulation [following a 50-ms recovery period (postLED)] was used to quantify stimulation-evoked desynchronized events ( $\mathrm{Sr}^{2+}$ mEPSCs) because of the presence of $\mathrm{SrCl}_{2}$ and absence of $\mathrm{CaCl}_{2}$. To calculate the amplitude of $\mathrm{Sr}^{2+} \mathrm{mEPSC}$ evoked by stimulation we used the following equation: [(postLED amplitude $\times$ postLED frequency) - (preLED amplitude $\times$ preLED frequency)]/(postLED frequency - preLED frequency). Cells were only used if the postLED frequency was at least $2 \mathrm{~Hz}$ higher than preLED frequency. Cell properties are listed in Extended Data Figure 3-1.

\section{Excitatory/inhibition balance}

rAAV-labeled L5 neurons were patched for whole-cell recordings in voltage clamp configuration in normal ACSF with $100 \mu \mathrm{M}$ APV added to block NMDA receptors. Callosally mediated EPSCs were elicited with LED stimulation of ChR2 transfected terminals arising from the contralateral virally transfected S1BC. LED intensity was adjusted to evoke similar sized EPSCs in sham versus ION-X neurons for each output neuron type. After stable sized events were achieved 20 repetitions were recorded from each neuron, switching between $-70 \mathrm{mV}$ for EPSCs and $0 \mathrm{mV}$ for IPSCs.

\section{Input/output (I/O) curves}

rAAV-labeled L5 neurons were patched for current-clamp recordings and LED stimulation of ChR2-expressing CC terminals was used to verify CC targeting. Electrical stimulation was used to measure the I/O curve, via a bipolar electrode (FHC) controlled by a DS3 isolated constant current stimulator (Digitimer Ltd.). The stimulating electrode was placed 1-1.6 mm medial to the recorded L5 neurons to avoid thalamocortical (TC) activation into the cortical column. A reliable monosynaptic response ( $<2$-ms onset latency) with similar kinetics to that evoked by LED activation of ChR2 expressing callosal terminals was achieved for each cell. The maximum amplitude response to intensity was measured as $100 \%$ response, and subsequent stimulus intensities were decreased to: $80 \%, 60 \%, 40 \%, 20 \%$, and $10 \%$ of the $100 \%$ stimulus intensity. Rarely a spike was elicited using this method, in those cases the $100 \%$ was labeled as the stimulus intensity immediately below spike threshold. There were no significant levels of spiking detected from output neurons in sham or after ION-X. The slope was calculated for I/O curves between $20 \%$ and $80 \%$ points to avoid the plateau affecting the dynamic range window's slope. Cell properties are listed in Extended Data Figure 4-1.

\section{Immunohistology and cell counting methods}

Two to three weeks after stereotaxic injection of one to four viruses, animals were anesthetized with $5 \%$ isoflurane vapor. Transcardial perfusion was performed with PBS followed by $4 \%$ paraformaldehyde and brains were removed. Cryopreserved and frozen brains expressing one to four fluorophores were sectioned at $30 \mu \mathrm{m}$ thick on a cryostat (Leica CM3050 S) and mounted onto glass slides. Sections were coverslipped using ProLong Gold antifade reagent (Fisher Scientific). Slides were imaged on a Zeiss Axioscan.Z1. Spectral unmixing was performed using Zeiss ZEN to eliminate bleed-through between emission spectra from the four fluorescent proteins: GFP, mOrange, mAmetrine, and mCherry. Sections from animals injected with a single virus were imaged using the same parameters as the four-color samples, and a reference spectrum was selected for each chromophore. Before cell counting, each multi-injection section was spectrally unmixed using the reference spectra. Individual cell bodies for each channel were identified using the cell detection feature in NeuroInfo (MBF Bioscience). For each image, cell size and intensity filter settings were adjusted to identify rAAV-labeled cells. Individual sections were registered to the Allen Mouse Brain Atlas using NeuroInfo and labeled cells were counted in specific barrel field layers of the deprived S1BC.

\section{Statistics}

Statistical tests compared the differences between values from single cells (n). Comparison across two groups were performed with Student's $t$ test and the Wilcoxon rank-sum test. To compare between groups of output neurons (i.e., sham S1BC vs M1, S2, and ACC) a two-way ANOVA 
A

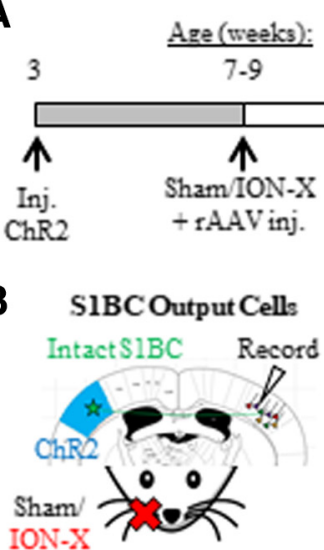

C

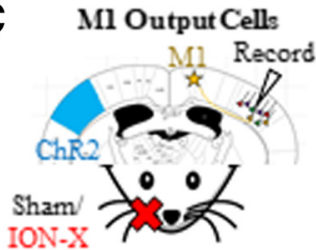

D

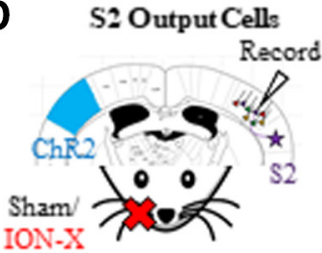

E

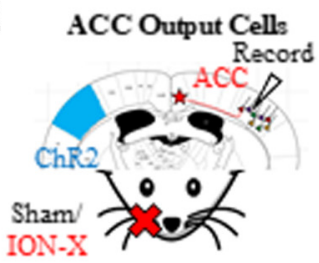

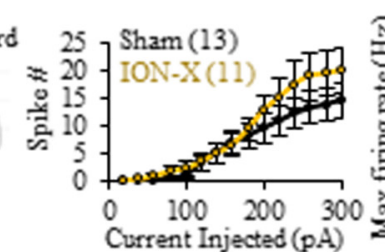

Current Injected (pA)
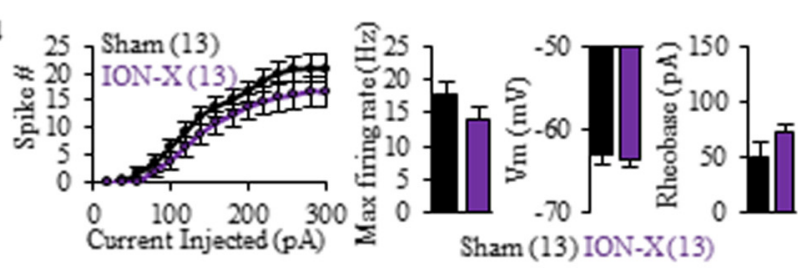
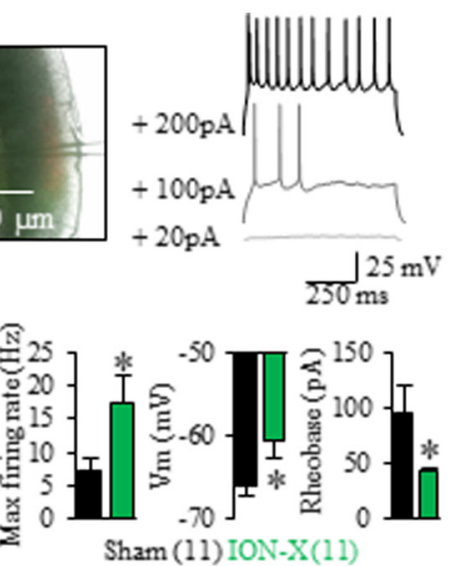

regions, including: intact S1BC, S2, M1, or ACC (Fig. 1A, left). Two weeks after ION$\mathrm{X}$ was determined to be the maximal time point for plasticity along the intact pathway (Yu et al., 2012; Chung et al., 2017), and robust callosal alterations were also described at this time point ( $\mathrm{Yu}$ and Koretsky, 2014; Petrus et al., 2019). Therefore, acute slices were made two weeks after ION-X, and rAAV-labeled cells were targeted for whole-cell current-clamp recordings. Responses to $\mathrm{ChR} 2$ were first verified to ensure only CC-targeted cells were studied. After wash-on with drugs to block synaptic activity, intrinsic neuronal properties were measured, including depolarization-induced spiking, resting membrane potential $(\mathrm{Vm})$, and rheobase values (Fig. 1A, right). Deprived L5 neurons reciprocally connected to intact S1BC exhibited increased maximum spike rate, a depolarized $\mathrm{Vm}$ and a lower rheobase. Student's $t$ test for sham versus ION-X $p=0.036, p=0.044$, and $p=$ 0.038 , respectively (Fig. $1 B$; Extended Data Fig. 1-1). Deprived L5 neurons projecting to $\mathrm{M} 1$ also experienced depolarized Vm and lower rheobase values but did not exhibit altered spiking properties. Student's $t$ test for sham versus ION-X $p=0.025, p=0.043$, and $p=$ 0.467, respectively (Fig. 1C; Extended Data Fig. 1-1). S2 output cells did not exhibit significant changes in any parameters measured. Student's $t$ test for sham versus ION-X $p=0.182, p=0.712$, and $p=0.161$ for spike rate, $\mathrm{Vm}$, and rheobase values (Fig. $1 D$; Extended Data Fig. 1-1), while ACC output cells experienced a decrease in maximum firing rate but no change in $\mathrm{Vm}$ or rheobase. Student's $t$ test for sham versus ION-X $p$ $=0.042, p=0.203$, and $p=0.136$, respectively (Fig. 1E; Extended Data Fig. 1-1). Overall, these results indicate that CCtargeted L5 output cells experience a heterogenous and output-region-specific adaptation to loss of sensory drive in deprived S1BC.

followed by Tukey's multiple comparisons test was used. For all tests, $p<0.05$ was considered statistically significant. Effect size was calculated using Cohen's $d$ to compare the distributions of means; values accompany the extended data figures for datasets.

\section{Results}

S1BC and M1 output cells are hyperexcitable after ION-X Unilateral whisker denervation increased excitability in CC-targeted L5 neurons in deprived S1BC (Petrus et al., 2019). Our hypothesis was that this change may be restricted to neurons with specific output targets. A virus encoding for channel rhodopsin (ChR2) was injected into intact S1BC, the infraorbital (whisker) nerve was transected (ION-X) and retrograde adeno-associated viruses (rAAVs) with a red fluorophore were injected into output

\section{Output cells are distinct populations}

Because the neurons studied here were all cortico-cortically projecting neurons, it has not been established if these are truly nonoverlapping groups of cells. Although others have described low levels of overlap between S2-projecting and M1-projecting neurons in S1 (Chakrabarti et al., 2008; Chen et al., 2013b), we sought to verify these findings and further describe the laminar profile of output neurons in S1BC. rAAVs expressing unique fluorophores were injected into the four output regions (Fig. 2A) and efficient separation between cell labeling was detected (Fig. $2 B)$. An Allen Brain Atlas was overlaid on the slices to detect the layers of S1BC (Fig. 2C, upper). Cells expressing more than one 
color were rarely seen $(<6 \%$ of any group; Fig. 2C, lower; Table 1). A large component of output cells was located in L5-6 regardless of cortical target (Fig. 2D; Table 1), further confirming that output neurons are located in deep cortical layers. Qualitatively, more cells with outputs to contralateral S1BC and M1 were detected than cells projecting to S2 and ACC (Table 1). The lack of overlap between cell groups and the location of labeling indicate that these output cells may represent an excellent mechanism by which the brain can direct both widespread and region-specific adaptations to changes in sensory experience.

\section{Callosal input is stronger to S1BC and} M1 output neurons but not S2 or ACC outputs

Previously it has been shown that callosal drive onto L5 neurons likely underlies bilateral cortical recruitment of S1BC after ION-X (Petrus et al., 2019). However, in the present study, we found that hyperexcitability was restricted to output-specific subgroups of L5 neurons that project to intact S1BC and M1 (Fig. 1). Therefore, to investigate possible output-specific changes in CC inputs onto these neurons, we substituted $\mathrm{Ca}^{2+}$ with $\mathrm{Sr}^{2+}$ in the extracellular fluid and CC-mediated events were recorded by stimulating ChR2-expressing axons arising from virally transfected contralateral neurons. The amplitudes of AMPA receptor mediated $\mathrm{Sr}^{2+}$ miniature EPSCs ( $\mathrm{Sr}^{2+}$ mEPSCs) were measured as a proxy for strength of the postsynaptic response to CC stimulation. After ION-X, the amplitude of postsynaptic responses to CC stimulation were larger in S1BC and M1 output cells; Student's $t$ test $p=0.006$ and $p=0.004$, respectively (Fig. $3 A, B$; Extended Data Fig. 3-1), while S2 and ACC output cells experienced no significant changes in strength; Student's $t$ test $p=0.423$ and $p=0.698$, respectively (Fig. 3C,D; Extended Data Fig. 3-1). These results demonstrate that both the intrinsic properties and postsynaptic potentiation are restricted to specific output cells after ION-X. The shift in excitability and synaptic strength may predispose CC-targeted S1BC and M1 output cells to respond to CC stimulation more readily than S2 or ACC neurons.

\section{Callosal release and evoked inhibition adaptations are restricted to S1BC output neurons}

The previous experiments determined that only S1BC and M1 output neurons experienced significant changes after ION-X, thus further investigation into these types of neurons was performed. S1BC and M1 output neurons experienced a change in both intrinsic properties and postsynaptic response to CC stimulation, it is possible that these changes were accompanied by presynaptic modifications of the CC. Paired pulse ratio (PPR) was measured in CC-targeted S1BC and M1 output cells after
ION-X. S1BC output cells had a small but significant shift toward paired pulse facilitation (PPF), indicating either an increase in CC release probability or, more likely, a reduction in callosally recruited inhibition (Fig. 4A; Extended Data Fig. 4-1, Student's $t$ test $p=0.028)$. The PPR was unchanged in M1 output neurons (Fig. 4B; Extended Data Fig. 4-1, Student's $t$ test $p=0.252$ ). This shift to PPF may also be explained by reduced inhibition which fails to "shunt" the second stimulus's excitatory response. Thus, we further studied how callosally evoked inhibition may be altered after ION-X.

Although the CC is a glutamatergic input (Kawaguchi, 1992; Conti and Manzoni, 1994; Petreanu et al., 2007), the net result of CC activity is usually inhibitory by providing strong input onto inhibitory neurons in the contralateral hemisphere. This is referred to as interhemispheric inhibition (IHI; Daskalakis et al., 2002; Palmer et al., 2012). We hypothesized that the shift toward responsiveness in S1BC and M1 output cells may be either compensated for by an increase or further emphasized by a decrease in CC-evoked inhibition. The CC-mediated excitation/inhibition 
Table 1. Numbers of cells counted expressing each retrograde AAV by injection location (color)

\begin{tabular}{|c|c|c|c|c|}
\hline$\overline{S 1 B C}$ (eGFP) & Sham $(6,1)$ & & ION-X $(5,1)$ & \\
\hline L1-3 & $56.5 \pm 8.3$ & & $41 \pm 20.5$ & \\
\hline L4 & $7.83 \pm 2.5$ & & $11.8 \pm 5.9$ & \\
\hline L5-6 & $79 \pm 10$ & & $79.8 \pm 39.9$ & \\
\hline Total & $143.3 \pm 58.5$ & & $132.5 \pm 66.3$ & \\
\hline M1 (m0range) & Sham $(6,1)$ & & ION-X $(7,2)$ & \\
\hline$\overline{\mathrm{L1}-3}$ & $88 \pm 39$ & & $35.3 \pm 13.3$ & \\
\hline L4 & $5.2 \pm 2.1$ & & $3.9 \pm 1.5$ & \\
\hline L5-6 & $55.5 \pm 22.7$ & & $30.1 \pm 11.4$ & \\
\hline Total & $148.7 \pm 60.7$ & & $69.3 \pm 26.2$ & \\
\hline S2 (mAmetrine) & Sham $(9,2)$ & & ION-X $(10,3)$ & \\
\hline$\overline{\mathrm{L1}-3}$ & $8.7 \pm 2.9$ & & $14.4 \pm 4.5$ & \\
\hline L4 & $0.6 \pm 0.2$ & & $1.5 \pm 0.5$ & \\
\hline L5-6 & $35.3 \pm 11.8$ & & $43.6 \pm 13.8$ & \\
\hline Total & $44.6 \pm 14.9$ & & $59.5 \pm 18.8$ & \\
\hline$\overline{\mathrm{ACC} \text { (mCherry) }}$ & Sham $(9,2)$ & & ION-X $(10,3)$ & \\
\hline$\overline{\mathrm{L1}-3}$ & $26.6 \pm 8.9$ & & $14.3 \pm 4.5$ & \\
\hline L4 & $1.9 \pm 0.6$ & & $0.3 \pm 0.1$ & \\
\hline L5-6 & $15.1 \pm 5.0$ & & $10.4 \pm 3.3$ & \\
\hline Total & $43.6 \pm 14.5$ & & $25 \pm 7.9$ & \\
\hline Colocalization & Sham $(6,1)$ & & ION-X $(4,1)$ & \\
\hline$\overline{S 1 B C}+M 1$ & $11.3 \pm 2.4$ & $3.8 \%$ & $2.8 \pm 1.8$ & $1.4 \%$ \\
\hline $\mathrm{S} 1 \mathrm{BC}+\mathrm{S} 2$ & $1.5 \pm 0.5$ & $0.8 \%$ & $11.5 \pm 6.6$ & $6.0 \%$ \\
\hline $\mathrm{S} 1 \mathrm{BC}+\mathrm{ACC}$ & $3.0 \pm 0.7$ & $1.6 \%$ & $0 \pm 0$ & $0 \%$ \\
\hline$M 1+S 2$ & $3.2 \pm 0.9$ & $1.6 \%$ & $3.8 \pm 3.1$ & $2.9 \%$ \\
\hline $\mathrm{M} 1+\mathrm{ACC}$ & $6.0 \pm 1.5$ & $3.1 \%$ & $4.0 \pm 2.0$ & $4.3 \%$ \\
\hline $\mathrm{S} 2+\mathrm{ACC}$ & $0.5 \pm 0.3$ & $0.6 \%$ & $0.3 \pm 0.3$ & $0.3 \%$ \\
\hline
\end{tabular}

$(n, n)$ reflects number of slices, animals. Values are mean \pm SEM. Colocalization indicates the number of cells expressing more than one color by injection site, percentages are calculated by number of cells expressing both fluorophores/sum number of cells expressing single fluorophores of both colors. There were no statistically significant differences in numbers of cells for each group of output cells between sham versus ION-X animals.

(E/I) ratio was measured in rAAV-labeled neurons held at $-70 \mathrm{mV}$ to evoke normalized excitatory AMPA events and $0 \mathrm{mV}$ for inhibitory GABA events. As expected, strong inhibition was observed in both sham groups as the measured $\mathrm{E} / \mathrm{I}$ ratio was $<1$ (Fig. 4C,D). However, after ION-X CC-evoked inhibition was reduced to reciprocally connected $\mathrm{S} 1 \mathrm{BC}$ output neurons with an increase in the E/I ratio to $>1.5$; Student's $t$ test $p=0.043$ (Fig. 4C; Extended Data Fig. 4-1). The M1 output cells had no significant change in E/I ratio; Student's $t$ test $p=0.175$ (Fig. $4 D$; Extended Data Fig. 4-1). Callosal excitation was larger as measured by larger AMPA receptor mediated $\mathrm{Sr}^{2+}$ mEPSCs (Fig. $3 B$ ), thus the CC-mediated inhibition may increase to match the excitation in M1 output neurons.

Because $\mathrm{PV}^{+}$cells are known to be modified by experience and exert strong perisomatic inhibition (Jiang et al., 2005; Donato et al., 2013), it was hypothesized that there may be a change in $\mathrm{CC}$ targeting of $\mathrm{PV}^{+}$interneurons. However, there was no significant change in the amplitude of CC-mediated $\mathrm{Sr}^{2+}$ mEPSCs onto $\mathrm{PV}^{+}$interneurons; Student's $t$ test $p=0.957$ (Fig. 4E; Extended Data Fig. 4-1).

Overall, these results indicate that neurons undergo outputtarget-specific adaptations in response to changes in sensory experience, with the cells projecting from deprived S1BC back to the intact S1BC experiencing the largest changes. These adaptations may shift the state of reciprocally connected neurons to be more responsive to $\mathrm{CC}$ input after ION-X compared with other projecting cells.

S1BC-projecting neurons have increased output responses to columnar inputs

After ION-X, deprived S1BC output neurons are hyperexcitable (Fig. 1), have stronger responses to CC stimulation from the
A
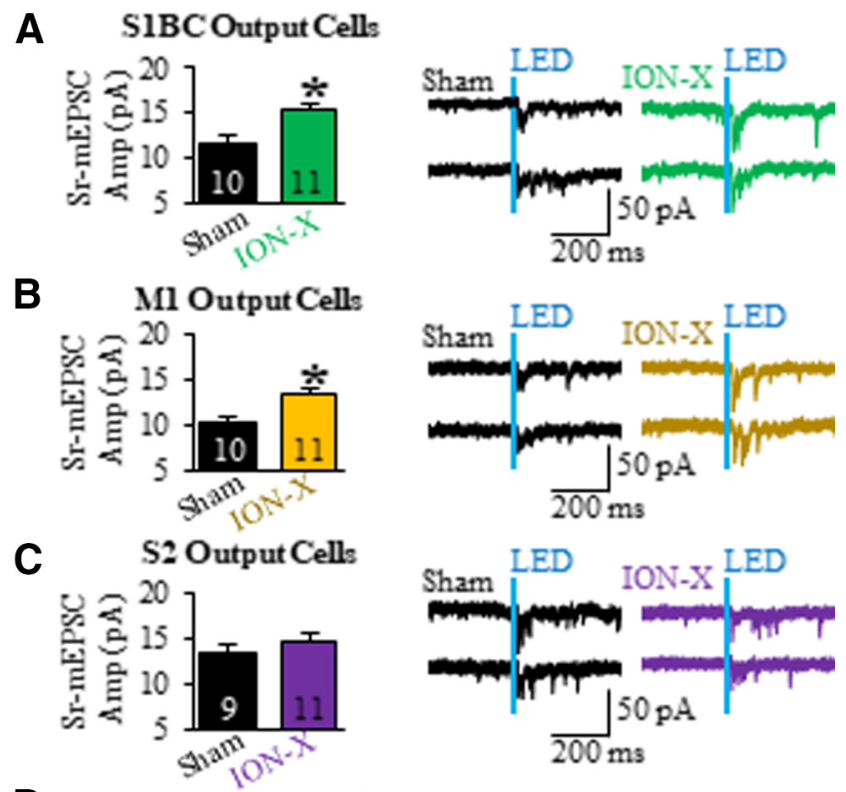

D ACC Output Cells
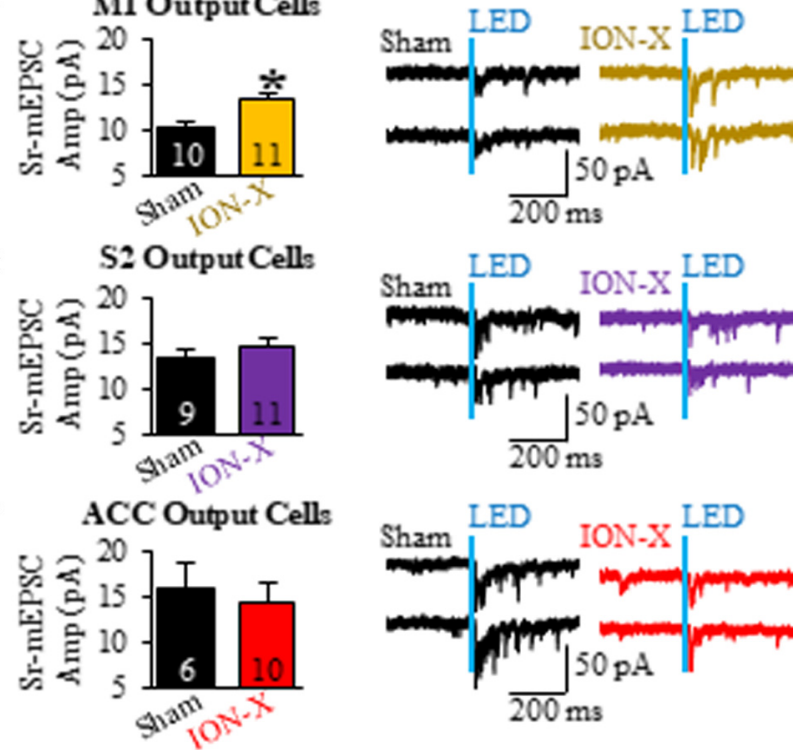

Figure 3. Callosal input is stronger to S1BC and M1 output neurons but not S2 or ACC outputs. Output neurons were patched and $\mathrm{Sr}^{2+}$ mEPSCs were recorded in response to LED stimulation of ChR2-expressing CC fibers. $\boldsymbol{A}, \boldsymbol{B}$, After ION-X, S1BC and M1 output neurons had larger amplitude $S^{2+}$ mEPSCS. C, D, S2 and ACC output neurons were not significantly altered. $(n)=$ number of cells, ${ }^{*} p<0.05 t$ test; for values, see Extended Data Figure 3-1.

intact S1BC, and are less inhibited by the CC (Figs. 3, 4). This led to the hypothesis that the efficacy of callosal transmission to this group of neurons may be increased compared to other output neurons. rAAV-labeled neurons were targeted for whole-cell current-clamp recordings and responses to ChR2 transfected callosally projecting neurons were verified with LED stimulation. Optogenetically evoked responses are known to be unstable at lower intensity stimuli (Morales et al., 2002; Bridi et al., 2020), so $\mathrm{I} / \mathrm{O}$ curves were generated with electrical stimulation. A bipolar electrode was placed on CC fibers medial to the recorded neuron to evoke events with more stable electrical stimulation. These events were monosynaptic and with similar kinetics to those evoked by LED stimulation of ChR2 expressing callosal terminals. The medial location was chosen to avoid activation of TC fibers; however, we cannot rule out the possibility that antidromic or non-callosal white matter inputs were stimulated by the electrodes. Regardless, we aimed to use the I/O curve as a rough estimate of how different output neurons respond to stimulation of callosal inputs to the cortical column. The maximum response size was found per cell and subsequent stimuli intensity were decreased by regular intervals (Fig. 5A). After ION-X, S1BC output neurons have a steeper I/O slope (Fig. 5C; Extended Data Fig. 5-1), while no change was found after ION-X in neurons projecting to M1, S2, or ACC. Student's $t$ test $p=0.032, p=0.603$, $p=0.618$, and $p=0.341$, respectively (Fig. $5 D-F$; Extended Data Fig. 5-1). The cumulative changes in S1BC output neurons may cooperate to produce a larger response to CC stimulation compared with other output cells. 
A

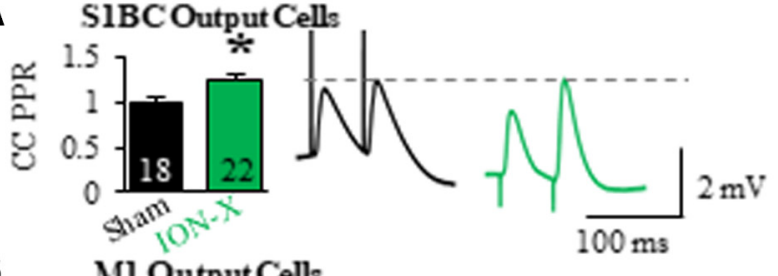

B Ml Output Cells

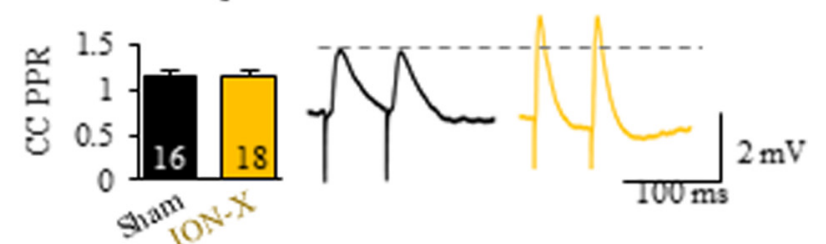

C S1BCOutput Cells
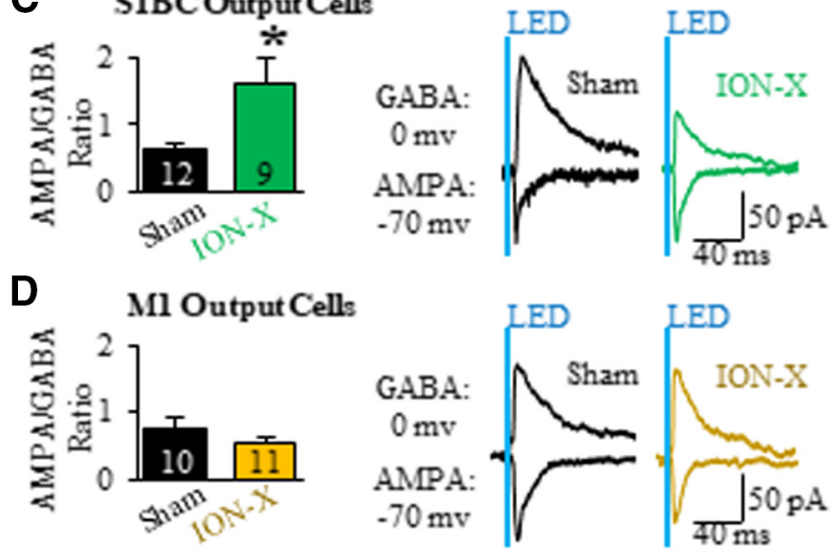

E
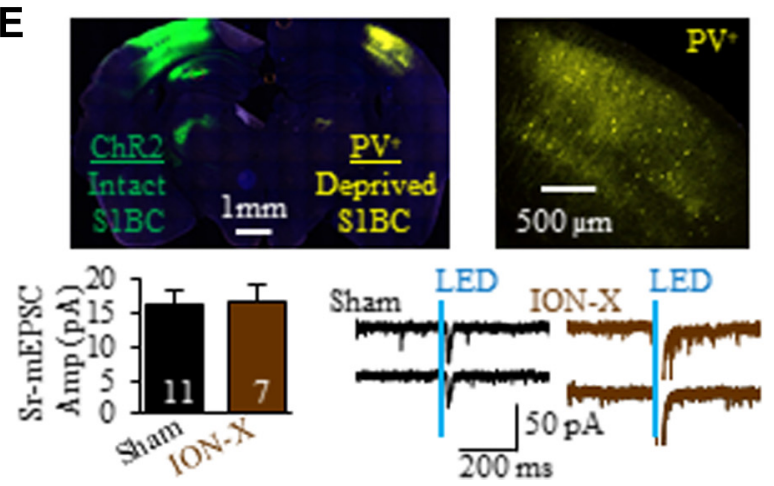

Figure 4. Callosal release and evoked inhibition adaptations are restricted to S1BC output neurons. $A, B$, PPR was measured to detect changes in callosal recruitment of inhibition or release probability onto S1BC and M1 output neurons. After ION-X, PPR shifted to facilitation at $C C$ synapses to $S 1 B C$ output neurons. CC synapses to $M 1$ output neurons were unchanged after ION-X. C, D, E/I (AMPA/GABA) ratio was measured in S1BC and M1 output neurons in response to $C($ stimulation. S1BC output neurons had significantly less inhibition after ION-X, while M1 inhibition matches excitation. $\boldsymbol{E}$, top, Expression of green ChR2 and yellow $\mathrm{PV}^{+}$ neurons in S1BC. Bottom, after ION-X CC-mediated $\mathrm{Sr}^{2+} \mathrm{mEPSCS}$ have no change in amplitude to $\mathrm{PV}^{+}$neurons. $(n)=$ number of cells, ${ }^{*} p<0.05 t$ test; for values, see Extended Data Figure 4-1.

A summary of the expected changes in circuit dynamics is depicted in Figure $5 B$. Note the increased intrinsic excitability and stronger CC-mediated synapses in S1BC and M1 output cells $(+)$, and reduction in inhibition only in S1BC output neurons (-). These factors produce a stronger response to CC stimulation only in cells that project back to intact S1BC. This increased response in reciprocally connected S1BC neurons may produce increased bilateral connectivity; denoted by a larger arrow out of the cortical column projecting to contralateral S1BC.

\section{Discussion}

The goal of this study was to determine whether the brain can restrict experience-dependent plasticity to specific groups of output neurons. Unilateral whisker denervation (ION-X) produced robust and specific adaptations in deprived neurons targeted by the CC. Brain regions were chosen based on their connectivity with the somatomotor system (intact S1BC, M1, S2) or their function in pain perception (S2, ACC). Reciprocally connected S1BC L5 neurons in deprived S1BC experienced the most robust shift to enhance responsiveness to CC stimulation. M1 output neurons experienced a similar shift toward increased CC responsiveness but a concurrent increase in inhibition negated more dramatic change in callosal responses. No significant changes after ION-X were detected in cells projecting to S2 and ACC. These results support the hypothesis that the recruitment of deprived S1BC is to increase bilateral cortical responsiveness to intact whisker stimulation. This recruitment is thus primed to interact with the intact S1BC during whisker sensation.

Peripheral loss of sensation alters the way the brain processes sensory information, leading to beneficial or maladaptive consequences. In particular, a unilateral loss unbalances the competitive bilateral nature of sensation, which leads to reorganization in adults (Finnerty and Connors, 2000). Along the intact TC pathway, critical period-like plasticity can be reopened to increase responses after sensory deprivation (Montey and Quinlan, 2011; Petrus et al., 2014; Chung et al., 2017; Hensch and Quinlan, 2018). Deprived TC pathways can remodel quickly (Coleman et al., 2010) or dieback after longer deprivations (Van der Loos and Woolsey, 1973; Oberlaender et al., 2012). In addition, bilateral cortical responses are observed with intact movement or sensation (Lotze et al., 2001; Pelled et al., 2007; Simões et al., 2012; Yu et al., 2012; Sammons and Keck, 2015). The purpose of this recruitment remains a puzzle: does it increase the brain processing power to enhance sensitivity to intact senses? Does it create a hyperexcitable region which by its mis-activation impairs recovery or causes pain? In the model of unilateral denervation studied in the present work, the lack of change in output cells targeting pain perception areas (S2, ACC) suggest that the adaptations are not causing a pain phenotype, but instead increasing the reciprocal connectivity between interhemispheric S1BCs.

Output S1BC neurons are distinct populations with unique characteristics as described here and by others (Hattox and Nelson, 2007; Chakrabarti et al., 2008; Chen et al., 2013b; Oswald et al., 2013; Kinnischtzke et al., 2016). Previous reports have described cortico-cortically projecting L5 neurons as a homogenous group. Here basal differences in neuronal properties were apparent indicating that this is a heterogeneous group. For example, the maximum firing rate, strength of response to the $\mathrm{CC}$ and $\mathrm{I} / \mathrm{O}$ properties in sham animals differed significantly depending on the target of S1BC output neurons (Figs. 1, 3, 4), and after ION-X more robust alterations were observed. Outputspecific adaptations are believed to be recruited to learn new behaviors. For example, accurate whisker task performance increased depolarizations in S1 neurons projecting to S2 but not M1 (Chen et al., 2013b; Yamashita and Petersen, 2016). The cellular/synaptic mechanisms of these results are not known. These output-specific modifications may be a way the cortex restricts changes to crucial neurons rather than the entire layer and all its outputs, in response to a specific behavioral requirement (Adesnik and Naka, 2018).

The adaptations of reciprocally connected S1BC neurons measured in the present study include a shift toward hyper- 
A

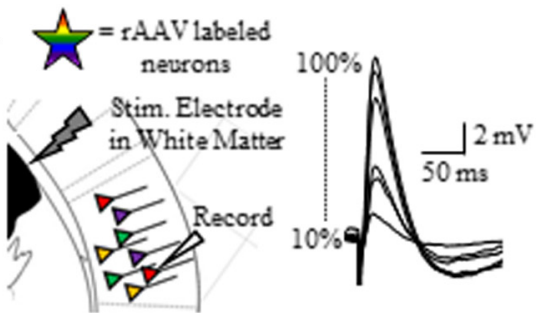

C S1BCOutputCells

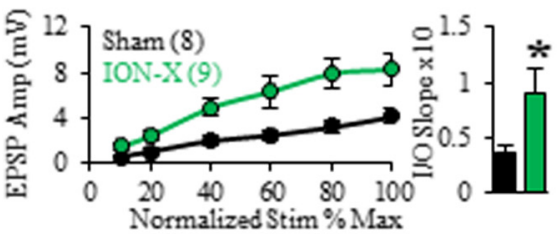

E S2 Output Cells

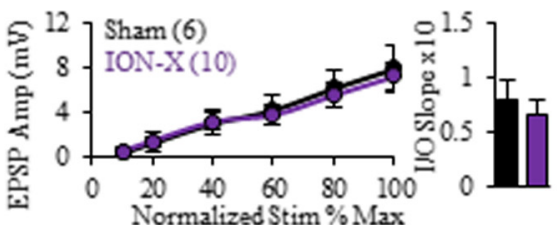

B

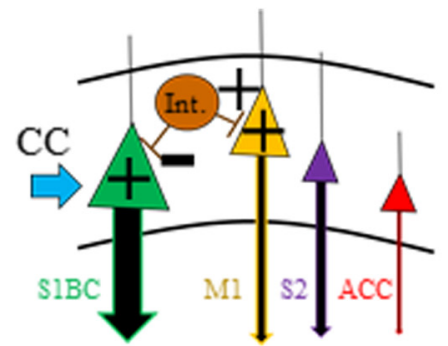

D Ml Output Cells

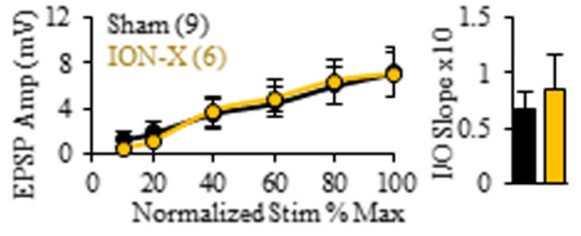

\section{F ACC Output Cells}

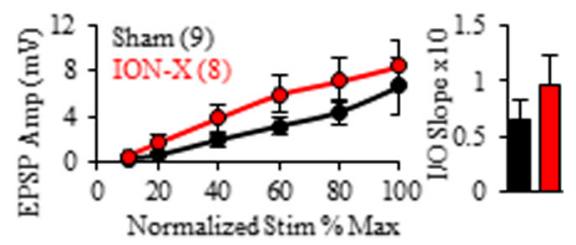

Figure 5. S1BC-projecting neurons have increased output responses to columnar inputs. $\boldsymbol{A}$, Experimental setup with bipolar electrode stimulating CC fibers medial to the rAAV-labeled output neuron. I/0 curves were generated for each output cell type, with responses recorded at regular intervals from the maximum response stimulus intensity. $\boldsymbol{B}$, Summary diagram of detected changes in deprived S1BC after ION-X. C-F, After ION-X, S1BC output neurons have steeper I/O curve slope, while other output groups are not significantly altered. $(n)=$ number of cells, ${ }^{*} p<0.05 t$ test; for values, see Extended Data Figure 5-1.

excitability, and a stronger postsynaptic response to CC inputs; these results are consistent with our previous study (Petrus et al., 2019). Hyperexcitability is found clinically in deprived cortex after amputation or subcortical stroke (Ziemann et al., 1998; Chen et al., 2002; Sammons and Keck, 2015). This increased activity in deprived somatomotor areas is especially pronounced two weeks after injury and predicts poor motor outcomes in stroke patients (Rehme et al., 2011). Increased excitability allows neurons to modify throughput capacity, meaning a more excitable cell is more likely to pass along presynaptic input as a postsynaptic output (Zhang and Linden, 2003). In addition, hyperexcitability may make long-term plasticity mechanisms like LTP more likely to occur at specific synapses (Moyer et al., 1996). Although the deprived cortex may globally experience less activity after removal of principal whisker input, callosal synapses onto S1BC and M1 output neurons were strengthened. Stronger postsynaptic CC responses were mediated by increased AMPA receptor activity, a hallmark of LTP (Malinow and Malenka, 2002). Reciprocally connected S1BC and M1 output neurons may combine this hyperexcitability and stronger CC responses to create a sensory processing situation such that the intact whisker pathway is more likely to evoke downstream activity only to M1 and back and forth along the CC between intact and deprived S1BCs.

IHI is the inhibitory influence of bilateral cortices on each other's activity (Chiarello and Maxfield, 1996). Activity in one sensorimotor cortex inhibits activity in the contralateral hemisphere, likely through excitatory CC synapses onto interneurons, which then inhibit local circuitry (Chen et al., 2002; Palmer et al., 2013). Consistent with this function, sham animals experienced larger inhibitory than excitatory events evoked by CC stimulation, but after ION-X, inhibition was reduced onto reciprocally connected S1BC neurons and remained strong for M1 output neurons (Fig. 4). Indeed, this reduction of inhibition onto reciprocally connected S1BC neurons may have reduced GABAergic shunting of callosal inputs, resulting in a shift toward PPF in S1BC, but not M1, output neurons (Fig. 4). Although the amplitude of CC-mediated $\mathrm{Sr}^{2+}$ mEPSCs was unchanged onto $\mathrm{PV}^{+}$interneurons, it is likely another group of interneurons play a more important role in IHI. It has been demonstrated that interneurons in L1, likely neurogliaform cells, produce IHI via $\mathrm{GABA}_{\mathrm{B}}$ receptors on the apical dendrites of L5 pyramidal cells (Palmer et al., 2012). Further study would be needed to determine whether this reduction in IHI between intact and deprived S1BCs is indeed mediated by activity in this subgroup of L1 interneurons. GABAergic interneurons help network pyramidal neurons decide when to fire (Pouille and Scanziani, 2001; Wehr and Zador, 2003) and increase their firing rates during tasks (Cardin et al., 2009; Sohal et al., 2009). These functions reduce pyramidal firing and fine tune their output to other brain regions (D'Souza and Burkhalter, 2017; Petersen, 2019).

The reduced inhibition onto reciprocally connected S1BC L5 neurons and increase of inhibition onto MI projecting cells may be a way for the deprived cortex to enhance direct reciprocal connectivity without affecting intrahemispheric outputs.

The increased direct reciprocal connectivity after ION-X likely has a systems level function. One purpose of the CC is to integrate unilateral stimuli into a complete representation of the external world (Pietrasanta et al., 2012). To do this, it must target excitatory and inhibitory neurons (Toyama and Matsunami, 1976; Payne and Siwek, 1991; Makarov et al., 2008; Rochefort et al., 2009; Palmer et al., 2013) to shape receptive fields (Watroba et al., 2001), accurately tune to salient stimuli (Rock and Apicella, 2015) and coordinate appropriate outputs; the CC often targets L5 neurons to achieve these results (Shuler et al., 2001, 2002). What would be the purpose of increasing the direct reciprocal connectivity? There have been three proposals for the role of the takeover of deprived cortex. One is to increase cortical territory to aid in processing. The most dramatic examples of this phenomenon is language re-lateralization after damage to the dominant hemisphere (Cao et al., 1999; Finger et al., 2003) and somatosensory processes using the visual cortex for processing in blind people (Cohen et al., 1997; Merabet et al., 2008). A clear reason for the takeover after unilateral sensory loss has not been established. However, the fact that the largest effect detected in the present study underlies the cortices ability to send information between the two sensory hemispheres is consistent with an adaptive takeover hypothesis. A second proposal may be that the activation of the deprived cortex is maladaptive and plays a role in 
phantom limb or prosthesis rejection. The lack of plasticity to pain pathways (S2 or ACC) argues against this in this model of denervation induced plasticity. Finally, it has been proposed that the recruitment of deprived cortex may help protect this deprived cortex from takeover from neighboring somatosensory regions like those that respond to nose or forepaw stimuli (Pluto et al., 2005; Yu and Koretsky, 2014). Such a model might require an ongoing callosal signal to report on the status of the deprived cortex. It is unknown whether these circuit level adaptations have a behavioral output, but these results are valuable to guide future studies in models of peripheral injury. Here, we have demonstrated that unilateral denervation alters cortical circuitry with a high degree of specificity to enhance bilateral responsiveness to intact senses.

\section{References}

Adesnik H, Naka A (2018) Cracking the function of layers in the sensory cortex. Neuron 100:1028-1043.

Baker A, Kalmbach B, Morishima M, Kim J, Juavinett A, Li N, Dembrow N (2018) Specialized subpopulations of deep-layer pyramidal neurons in the neocortex: bridging cellular properties to functional consequences. J Neurosci 38:5441-5455.

Bridi MCD, Zong FJ, Min X, Luo N, Tran T, Qiu J, Severin D, Zhang XT, Wang G, Zhu ZJ, He KW, Kirkwood A (2020) Daily oscillation of the excitation-inhibition balance in visual cortical circuits. Neuron 105:621629.

Cao Y, Vikingstad EM, George KP, Johnson AF, Welch KMA (1999) Cortical language activation in stroke patients recovering from aphasia with functional MRI. Stroke 30:2331-2340.

Cardin JA, Carlén M, Meletis K, Knoblich U, Zhang F, Deisseroth K, Tsai LH, Moore CI (2009) Driving fast-spiking cells induces gamma rhythm and controls sensory responses. Nature 459:663-667.

Chakrabarti S, Zhang M, Alloway KD (2008) MI neuronal responses to peripheral whisker stimulation: relationship to neuronal activity in SI barrels and septa. J Neurophysiol 100:50-63.

Chen R, Cohen LG, Hallett M (2002) Nervous system reorganization following injury. Neuroscience 111:761-773.

Chen A, Yao J, Kuiken T, Dewald JPA (2013a) Cortical motor activity and reorganization following upper-limb amputation and subsequent targeted reinnervation. Neuroimage Clin 3:498-506.

Chen JL, Carta S, Soldado-Magraner J, Schneider BL, Helmchen F (2013b) Behaviour-dependent recruitment of long-range projection neurons in somatosensory cortex. Nature 499:336-340.

Chiarello C, Maxfield L (1996) Varieties of interhemispheric inhibition, or how to keep a good hemisphere down. Brain Cogn 30:81-108.

Chung S, Jeong JH, Ko S, Yu X, Kim YH, Isaac JTR, Koretsky AP (2017) Peripheral sensory deprivation restores critical-period-like plasticity to adult somatosensory thalamocortical inputs. Cell Rep 19:2707-2717.

Clare AJ, Day RC, Empson RM, Hughes SM (2018) Transcriptome profiling of layer 5 intratelencephalic projection neurons from the mature mouse motor cortex. Front Mol Neurosci 11:410-414.

Cohen LG, Celnik P, Pascual-Leone A, Corwell B, Falz L, Dambrosia J, Honda M, Sadato N, Gerloff C, Catalá MD, Hallett M (1997) Functional relevance of cross-modal plasticity in blind humans. Nature 389:180-183.

Coleman JE, Nahmani M, Gavornik JP, Haslinger R, Heynen AJ, Erisir A, Bear MF (2010) Rapid structural remodeling of thalamocortical synapses parallels experience-dependent functional plasticity in mouse primary visual cortex. J Neurosci 30:9670-9682.

Conti F, Manzoni T (1994) The neurotransmitters and postsynaptic actions of callosally projecting neurons. Behav Brain Res 64:37-53.

Daskalakis ZJ, Christensen BK, Fitzgerald PB, Roshan L, Chen R (2002) The mechanisms of interhemispheric inhibition in the human motor cortex. J Physiol 543:317-326.

Donato F, Rompani SB, Caroni P (2013) Parvalbumin-expressing basket-cell network plasticity induced by experience regulates adult learning. Nature 504:272-276.

Douglas RJ, Martin KAC (2004) Neuronal circuits of the neocortex. Annu Rev Neurosci 27:419-451.

D'Souza RD, Burkhalter A (2017) A laminar organization for selective cortico-cortical communication. Front Neuroanat 11:71.
Feldmeyer D (2012) Excitatory neuronal connectivity in the barrel cortex. Front Neuroanat 6:24-22.

Finger S, Buckner R, Buckingham H (2003) Does the right hemisphere take over after damage to Broca's area? the Barlow case of 1877 and its history. Brain Lang 85:385-395.

Finnerty GT, Connors BW (2000) Sensory deprivation without competition yields modest alterations of short-term synaptic dynamics. Proc Natl Acad Sci USA 97:12864-12868.

Flor H, Nikolajsen L, Jensen TS (2006) Phantom limb pain: a case of maladaptive CNS plasticity? Nat Rev Neurosci 7:873-881.

Garraghty PE, Kaas JH (1991) Large-scale functional reorganization in adult monkey cortex after peripheral nerve injury. Proc Natl Acad Sci USA 88:6976-6980.

Grillner S, Markram H, De Schutter E, Silberberg G, LeBeau FEN (2005) Microcircuits in action - from CPGs to neocortex. Trends Neurosci 28:525-533.

Hattox AM, Nelson SB (2007) Layer V neurons in mouse cortex projecting to different targets have distinct physiological properties. J Neurophysiol 98:3330-3340.

Hensch TK, Quinlan EM (2018) Critical periods in amblyopia. Vis Neurosci 35:E014.

Jiang B, Huang ZJ, Morales B, Kirkwood A (2005) Maturation of GABAergic transmission and the timing of plasticity in visual cortex. Brain Res Brain Res Rev 50:126-133.

Kawaguchi Y (1992) Receptor subtypes involved in callosally-induced postsynaptic potentials in rat frontal agranular cortex in vitro. Exp Brain Res 88:33-40.

Kinnischtzke AK, Fanselow EE, Simons DJ (2016) Target-specific M1 inputs to infragranular S1 pyramidal neurons. J Neurophysiol 116:1261-1274.

Koester HJ, Johnston D (2005) Target cell-dependent normalization of transmitter release at neocortical synapses. Science 308:863-866.

Larsen RS, Sjöström PJ (2015) Synapse-type-specific plasticity in local circuits. Curr Opin Neurobiol 35:127-135.

Lee S, Zhang Y, Chen M, Zhou ZJ (2016) Segregated glycine-glutamate cotransmission from vGluT3 amacrine cells to contrast-suppressed and contrast-enhanced retinal circuits. Neuron 90:27-34

Lefort S, Petersen CCH (2017) Layer-dependent short-term synaptic plasticity between excitatory neurons in the $\mathrm{C} 2$ barrel column of mouse primary somatosensory cortex. Cereb Cortex 27:3869-3878.

Van der Loos H, Woolsey TA (1973) Somatosensory cortex: structural alterations following early injury to sense organs. Science 179:395-398.

Lotze M, Flor H, Grodd W, Larbig W, Birbaumer N (2001) Phantom movements and pain. An fMRI study in upper limb amputees. Brain 124:2268-2277.

Lu J, Tucciarone J, Lin Y, Huang ZJ (2014) Input-specific maturation of synaptic dynamics of parvalbumin interneurons in primary visual cortex. Proc Natl Acad Sci USA 111:16895-16900.

MacIver K, Lloyd DM, Kelly S, Roberts N, Nurmikko T (2008) Phantom limb pain, cortical reorganization and the therapeutic effect of mental imagery. Brain 131:2181-2191.

Makarov VA, Schmidt KE, Castellanos NP, Lopez-Aguado L, Innocenti GM (2008) Stimulus-dependent interaction between the visual areas 17 and 18 of the 2 hemispheres of the ferret (Mustela putorius). Cereb Cortex 18:1951-1960.

Makin TR, Scholz J, Filippini N, Henderson Slater D, Tracey I, JohansenBerg H (2013) Phantom pain is associated with preserved structure and function in the former hand area. Nat Commun 4:1570-1578.

Makin TR, Filippini N, Duff EP, Henderson Slater D, Tracey I, JohansenBerg H (2015) Network-level reorganisation of functional connectivity following arm amputation. Neuroimage 114:217-225.

Malinow R, Malenka RC (2002) AMPA receptor trafficking and synaptic plasticity. Annu Rev Neurosci 25:103-126.

Merabet LB, Hamilton R, Schlaug G, Swisher JD, Kiriakopoulos ET, Pitskel NB, Kauffman T, Pascual-Leone A (2008) Rapid and reversible recruitment of early visual cortex for touch. PLoS One 3:e3046.

Montey KL, Quinlan EM (2011) Recovery from chronic monocular deprivation following reactivation of thalamocortical plasticity by dark exposure. Nat Commun 2:317.

Morales B, Choi S-Y, Kirkwood A (2002) Dark rearing alters the development of GABAergic transmission in visual cortex. J Neurosci 22:80848090 . 
Moyer JR, Thompson LT, Disterhoft JF (1996) Trace eyeblink conditioning increases CA1 excitability in a transient and learning-specific manner. J Neurosci 16:5536-5546.

Navarro X, Vivó M, Valero-Cabré A (2007) Neural plasticity after peripheral nerve injury and regeneration. Prog Neurobiol 82:163-201.

Oberlaender M, Ramirez A, Bruno RM (2012) Sensory experience restructures thalamocortical axons during adulthood. Neuron 74:648-655.

Oswald MJ, Tantirigama MLS, Sonntag I, Hughes SM, Empson RM (2013) Diversity of layer 5 projection neurons in the mouse motor cortex. Front Cell Neurosci 7:174-118.

Palmer LM, Schulz JM, Murphy SC, Ledergerber D, Murayama M, Larkum ME (2012) Interhemispheric Inhibition. Science 335:989-993.

Palmer LM, Schulz JM, Larkum ME (2013) Layer-specific regulation of cortical neurons by interhemispheric inhibition. Commun. Integr. Biol 6:1-5.

Payne BR, Siwek DF (1991) The visual map in the corpus callosum of the cat. Cereb Cortex 1:173-188.

Pelled G, Chuang K-H, Dodd SJ, Koretsky AP (2007) Functional MRI detection of bilateral cortical reorganization in the rodent brain following peripheral nerve deafferentation. Neuroimage 37:262-273.

Petersen CCH (2019) Sensorimotor processing in the rodent barrel cortex. Nat Rev Neurosci 20:533-546.

Petreanu L, Huber D, Sobczyk A, Svoboda K (2007) Channelrhodopsin-2assisted circuit mapping of long-range callosal projections. Nat Neurosci 10:663-668.

Petrus E, Lee HK (2014) BACE1 is necessary for experience-dependent homeostatic synaptic plasticity in visual cortex. Neural Plast 2014:128631.

Petrus E, Isaiah A, Jones AP, Li D, Wang H, Lee H-K, Kanold PO (2014) Crossmodal induction of thalamocortical potentiation leads to enhanced information processing in the auditory cortex. Neuron 81:664-673.

Petrus E, Saar G, Ma Z, Dodd S, Isaac JTR, Koretsky AP (2019) Interhemispheric plasticity is mediated by maximal potentiation of callosal inputs. Proc Natl Acad Sci USA 116:6391-6396.

Pietrasanta M, Restani L, Caleo M (2012) The corpus callosum and the visual cortex: plasticity is a game for two. Neural Plast 2012:838672.

Pluto CP, Chiaia NL, Rhoades RW, Lane RD (2005) Reducing contralateral SI activity reveals hindlimb receptive fields in the SI forelimb-stump representation of neonatally amputated rats. J Neurophysiol 94:1727-1732.

Pouille F, Scanziani M (2001) Enforcement of temporal fidelity in pyramidal cells by somatic feed-forward inhibition. Science 293:1159-1163.

Rawson RL, Martin EA, Williams ME (2017) Mechanisms of input and output synaptic specificity: finding partners, building synapses, and fine-tuning communication. Curr Opin Neurobiol 45:139-148.

Rehme AK, Fink GR, Von Cramon DY, Grefkes C (2011) The role of the contralesional motor cortex for motor recovery in the early days after stroke assessed with longitudinal fMRI. Cereb Cortex 21:756-768.

Rochefort NL, Buzás P, Quenech'du N, Koza A, Eysel UT, Milleret C, Kisvárday ZF (2009) Functional selectivity of interhemispheric connections in cat visual cortex. Cereb Cortex 19:2451-2465.

Rock C, Apicella AJ (2015) Callosal projections drive neuronal-specific responses in the mouse auditory cortex. J Neurosci 35:6703-6713.

Sammons R, Keck T (2015) Adult plasticity and cortical reorganization after peripheral lesions. Curr Opin Neurobiol 35:136-141.
Shai AS, Anastassiou CA, Larkum ME, Koch C (2015) Physiology of layer 5 pyramidal neurons in mouse primary visual cortex: coincidence detection through bursting. PLoS Comput. Biol 11:1-18.

Shuler MG, Krupa DJ, Nicolelis MA (2001) Bilateral integration of whisker information in the primary somatosensory cortex of rats. J Neurosci 21:5251-5261

Shuler MG, Krupa DJ, Nicolelis MAL (2002) Integration of bilateral whisker stimuli in rats: role of the whisker barrel cortices. Cereb Cortex 12:86-97.

Simões EL, Bramati I, Rodrigues E, Franzoi A, Moll J, Lent R, Tovar-Moll F (2012) Functional expansion of sensorimotor representation and structural reorganization of callosal connections in lower limb amputees. J Neurosci 32:3211-3220.

Sjöström PJ, Häusser M (2006) A cooperative switch determines the sign of synaptic plasticity in distal dendrites of neocortical pyramidal neurons. Neuron 51:227-238

Sohal VS, Zhang F, Yizhar O, Deisseroth K (2009) Parvalbumin neurons and gamma rhythms enhance cortical circuit performance. Nature 459:698702.

Tasic B, Menon V, Nguyen TN, Kim TK, Jarsky T, Yao Z, Levi B, Gray LT, Sorensen SA, Dolbeare T, Bertagnolli D, Goldy J, Shapovalova N, Parry S, Lee C, Smith K, Bernard A, Madisen L, Sunkin SM, Hawrylycz M, et al. (2016) Adult mouse cortical cell taxonomy revealed by single cell transcriptomics. Nat Neurosci 19:335-346.

Templeton CA, Strzalkowski NDJ, Galvin P, Bent LR (2018) Cutaneous sensitivity in unilateral trans-tibial amputees. PLoS One 13:e0197557.

Toyama K, Matsunami K (1976) Convergence of specific visual and commissural impulses upon inhibitory interneurones in cats visual cortex. Neuroscience 1:107-112.

Trouche S, Sasaki JM, Tu T, Reijmers LG (2013) Fear extinction causes target-specific remodeling of perisomatic inhibitory synapses. Neuron 80:1054-1065

Watroba L, Buser P, Milleret C (2001) Impairment of binocular vision in the adult cat induces plastic changes in the callosal cortical map. Eur J Neurosci 14:1021-1029.

Wehr M, Zador AM (2003) Balanced inhibition underlies tuning and sharpens spike timing in auditory cortex. Nature 426:442-446.

Yamashita T, Petersen CCH (2016) Target-specific membrane potential dynamics of neocortical projection neurons during goal-directed behavior. Elife 5:e15798

Yu X, Koretsky AP (2014) Interhemispheric plasticity protects the deafferented somatosensory cortex from functional takeover after nerve injury. Brain Connect 4:709-717.

Yu X, Chung S, Chen DY, Wang S, Dodd SJ, Walters JR, Isaac JTR, Koretsky AP (2012) Thalamocortical inputs show post-critical-period plasticity. Neuron 74:731-742.

Zhang W, Linden DJ (2003) The other side of the engram: experience-driven changes in neuronal intrinsic excitability. Nat Rev Neurosci 4:885-900.

Ziemann U, Corwell B, Cohen LG (1998) Modulation of plasticity in human motor cortex after forearm ischemic nerve block. J Neurosci 18:11151123. 